\title{
BASES PARA UN PROGRAMA DE MEJORA DE ALPACAS EN LA REGION ALTOANDINADE HUANCAVELICA-PERÚ
}

\author{
BASES TO AN IMPROVEMENT PROGRAM OF THE ALPACAS IN HIGHLAND REGION AT \\ HUANCAVELICA-PERU
}

\author{
Quispe, E.C. ${ }^{1 *}$, L. Alfonso², A. Flores ${ }^{3}$, H. Guillén ${ }^{1}$ e Y. Ramos ${ }^{1}$
}

\begin{abstract}
1Departamento Académico de Zootecnia. Facultad de Ciencias de Ingeniería. Universidad Nacional de Huancavelica. Ciudad Universitaria Paturpampa s/n. Huancavelica. Perú. *edgarquispe62@yahoo.com 2Departamento de Producción Agraria. Universidad Pública de Navarra. Campus de Arrosadía 31006. Pamplona. España. leo.alfonso@unavarra.es

${ }^{3}$ Escuela de Postgrado. Universidad Nacional Agraria. Av. La Molina s/n. La Molina. Lima. Perú. adocejuna@unalm.edu.pe
\end{abstract}

\section{Palabras clave adicionales}

Lama pacos. Huacaya. Fibra. Esquema de mejora. Progreso genético.

\section{RESUMEN}

El estudio se desarrolló en 544 alpacas Huacaya de color blanco localizadas en 19 centros de producción de la Región de Huancavelica, ubicadas un rango de altitud entre 4000 y 4800 msnm. Antes de la esquila se tomaron muestras del vellón de aproximadamente $20 \mathrm{~g}$ de la zona del costillar y posterior a ella se registró el peso de vellón grasiento (PVG), sexo, edad y unidad productiva de cada animal. El diámetro de fibra (DF), coeficiente de variación del diámetro de fibra (CVDF) y el factor de picazón (FP) fue determinado utilizando el Sirolan-Laserscan. En función a los datos obtenidos se realizó el planteamiento de un esquema de mejora y sobre ello se estimó la respuesta a la selección bajo distintos escenarios. Los promedios $\pm E$.E. del PVG, DF, CVDF y FP fueron de $2300 \pm 39 \mathrm{~g}, 21,56 \pm 0,12 \mu \mathrm{m}, 22,82 \pm 0,12 \%$ y $6,33 \pm$ $0,30 \%$ respectivamente. EI PVG y el DF muestran variaciones significativas $(p<0,01)$ para sexo, edad y unidad productiva, mientras que el FP tiene variaciones significativas $(p<0,01)$ para edad y unidad de producción y el CVDF sólo muestra variaciones para unidad productiva $(p<0,01)$. Cuando fueron analizadas las relaciones entre las cuatro variables de la fibra, sólo se encontró significancia entre DF y FP, DF y PVG, CVDF y FP, y CVDF y DF. Bajo estas consideraciones el esquema de mejora planteado conduce a una

Recibido: 3-12-07. Aceptado: 17-11-08.

\section{AdDitional KEYWORDS}

Lama pacos. Huacaya. Fibre. Improvement scheme. Genetic gain.

formación de un núcleo élite de reproductores formados por 50 machos que podrían empadrar a las mejores 1100 hembras de los animales con registros. La predicción del progreso genético anual, en una selección masal se encuentra entre 57 y 78 g para PVG y entre $-0,17$ y $-0,23 \mu \mathrm{m}$ para DF; mientras que, en términos económicos el progreso genético anual usando un índice combinado se sitúa entre $\$ 0,83$ y $\$ 1,14$.

\section{SUMMARY}

The research was developed in 544 Huacaya alpacas of white color located in 19 centers of production of the Region of Huancavelica, located a rank of altitude between 4000 and 4800 meters above sea level. Before the shearing, approximately $20 \mathrm{~g}$ sample were taken from fleece of the mid side and after the shearing the grease fleece weight (PVG), sex, age and productive unit was registered of each animal. Fibre diameter (DF), coefficient of variation of fibre diameter (CVDF) and the prickle factor (FP) was determined using the Sirolan-Laserscan. In function to the collected data the exposition of an improvement scheme was made and on it we considered the answer to the selection under two scenarios. The means \pm S.E. of PVG, DF, CVDF and FP were of $2300 \pm 39$ 
g, $21.56 \pm 0.12 \mu \mathrm{m}, 22.82 \pm 0.12 \%$ and $6.33 \pm 0.30 \%$ respectively. Variation of $P V G$ and DF show significance $(p<0.01)$ for sex, age and productive unit, whereas variations of PF has significance $(p<0.01)$ for age and unit of production and variations of CVFD was only for productive unit $(p<0.01)$. When the relations between the four variables of the fibre were analyzed, only was significance between DF and FP, DF and PVG, CVDF and FP, and CVDF and DF. About these considerations the scheme of improvement leads to a formation of a nucleus elite of reproducers formed by 50 males who could mating to best the 1100 females of the animals with records. The annual genetic gain prediction, in an individual selection is between 57 and $78 \mathrm{~g}$ for GFW and -0.17 and $-0.23 \mu \mathrm{m}$ for FD; whereas, in economic values the annual genetic gain prediccion in a selection by index is between $\$ 0.83$ and $\$ 1.14$ depending if a pessimist or optimist scenario is considered.

\section{INTRODUCCION}

En América del Sur se estima que existen más de 7,5 millones de cabezas de camélidos sudamericanos, los cuales son agrupados en cuatros especies, y de ellos dos son silvestres: La vicuña (Vicugna vicugna) y el guanaco (Lama guanicoe); y dos son domésticos: La llama (Lama glama) y la alpaca (Lama pacos). En la actualidad, los camélidos sudamericanos probablemente constituyen el principal medio de utilización productiva de las extensas áreas de pastos naturales de las zonas alto andinas donde no es posible la agricultura ni la crianza económica de muchas especies de animales domésticos, debido a que convierten, con inusual eficiencia, los pastos pobres de estas alturas en productos de alta calidad como son la fibra y la carne. El Perú tiene el privilegio de ocupar el primer lugar en el mundo en la tenencia de alpacas y vicuñas y el segundo lugar en llamas, después de Bolivia. El aprovechamiento racional de esta ventaja comparativa es el reto que este país encara como el medio más efectivo de lucha contra la pobreza y la inseguridad alimentaria que afecta a las comunidades campesinas que viven de la crianza de estas especies.

La alpaca es el más importante miembro de los camélidos sudamericanos en cuanto se refiere a la producción de fibra (LeónVelarde y Guerrero, 2001; Wuliji et al., 2000), y en función a ella habría sido seleccionada desde hace más de 3000 años (Wang et al., 2003). La industria textil refiere a la fibra de alpaca como una fibra especial y las prendas que se confeccionan con ellas, están clasificadas como artículos de lujo (Wang et al., 2003). Su población mundial se estima en unos 3,7 millones (FAO, 2005) y el 80\% de ellas (aprox. 3 millones) se encuentran principalmente en las zonas alto andinas de Perú, de los que alrededor del $86 \%$ son alpacas de color blanco; y el remanente se ubica principalmente en Bolivia y Chile, aunque se han introducido también exitosamente en Australia, Canadá, Inglaterra, Francia, Nueva Zelanda y Estados Unidos (Lupton et al., 2006). Perú es el primer productor mundial de fibra de alpaca y en el año 2003 se registró una producción de 6440 t con un valor por encima de los 82 millones de dólares (De los Ríos, 2006).

Existen dos razas de alpacas: La Huacaya, que se caracteriza por ser compacta, de fibra suave y con presencia de ondulaciones, asimismo el vellón manifiesta una apariencia esponjosa, lo que le da una apariencia más voluminosa; y la Suri la cual presenta fibras de gran longitud que se organizan en rizos que caen por los costados del cuerpo, dándole al animal una apariencia angulosa (Hoffman y Fowler, 1995; Antonini et al., 2004; FAO, 2005). La raza Huacaya es la que predomina con un $85 \%$ mientras que la raza Suri sólo representa el 15\% de la población total.

En Huancavelica - región ubicada en una zona alto andina de la sierra centro de Perú - existen más de 225000 alpacas, las cuales constituyen alrededor del $7 \%$ de la población, y de ellas el $90 \%$ son de la raza Huacaya. La crianza de la alpaca constituye una importante actividad económica del poblador 


\section{PROGRAMA DE MEJORA DE ALPACAS EN HUANCAVELICA-PERÚ}

alto andino, la cual se desarrolla por encima de los $3700 \mathrm{msnm}$; las familias están organizadas en comunidades de pastores, bajo un sistema de crianza de Rebaño Mixto Familiar (alpacas, llamas, ovinos). Estos rebaños se caracterizan por carecer de sistemas de crianza adecuados, con escasos criterios de mejoramiento genético, basados exclusivamente como fuente alimenticia en pastizales alto andinos de baja soportabilidad, con escasez de cuidados sanitarios. Se estima en más de 60 las comunidades alpaqueras, que agrupan a 3300 familias aproximadamente; distribuidas principalmente en cuatro provincias: Angaraes, Huaytará, Castro-virreyna y Huancavelica (Quispe, 2005).

Los vellones obtenidos de las alpacas en las comunidades alpaqueras de Huancavelica se consideran de baja producción y calidad. A nivel de comunidades se estima una producción promedio bianual de $2,1 \mathrm{~kg}$, mientras que bajo una crianza medianamente tecnificada se obtiene una producción anual de 2,3 kg (Jáuregui y Bonilla, 1991; Nieto y Alejos, 1999). Con respecto a la finura De Los Ríos (2006), refiere que a nivel nacional, el $20 \%$ de la producción está dado por fibra gruesa (mayor de $29 \mu \mathrm{m}$ ), el $46 \%$ por fibra semi fina $(26,6$ a 29,0 $\mu \mathrm{m})$, el $22 \%$ por fibra fina $(23,1$ a $26,5 \mu \mathrm{m} \mathrm{s})$ y sólo el $12 \%$ está conformado por fibra extra fina (menor de $23,1 \mu \mathrm{m}$ ), lo cual vislumbra el enorme déficit en calidad.

Existen muchos estudios sobre fibra de alpaca, es así que se han realizado caracterizaciones de peso de vellón (Jáuregui y Bonilla, 1991; Mamani, 1991 y Frank et al., 2006); finura, longitud de fibra, medulación y otras características (León, 1998; Wang et al., 2003; Lupton et al., 2006 y Frank et al., 2006). Las relaciones entre peso de vellón, diámetro de fibra y otras características de importancia del vellón también han sido abordados por Ponzoni et al. (1999), Wuliji et al. (2000) y Wang et al. (2003). Los efectos tecnológicos y medioambientales fueron parcialmente determinados, en función a peso de vellón, finura y otros (Apomayta y Gutiérrez, 1998; León, 1998; Chávez y Pumayalla, 1988; McGregor y Butler, 2004; Lupton et al., 2006; Braga et al., 2006). La determinación de parámetros genéticos tales como la repetibilidad y heredabilidad para algunos caracteres de la fibra han sido tratados por Ponzoni et al. (1999) y Wuliji et al. (2000). Predicciones del progreso genético utilizando índices de selección fueron realizadas por Ponzoni (2000) y LeónVelarde y Guerrero (2001), quienes muestran ganancias genéticas referidas a peso de vellón, diámetro de fibra y longitud de mecha, bajo ciertos esquemas de selección.

Teniendo en consideración lo planteado en los párrafos anteriores se ha realizado el presente trabajo como línea de base de un programa de mejora de alpacas Huacaya de color Blanco en la Región de HuancavelicaPerú a fin de caracterizar el vellón (con respecto a peso de vellón, diámetro de fibra, coeficiente de variación del diámetro de fibra e índice de confort), identificar los efectos de sexo, edad y localidad sobre las características de la fibra, determinar la correlación fenotípica entre tales características y finalmente en base a dichos resultados estimar el progreso genético que se obtendría bajo diferentes escenarios, tomando en consideración los dos criterios de selección más importantes para la comercialización y la industria textil (el peso de vellón y el diámetro de fibra) y utilizando estimaciones de parámetros genéticos obtenidas de la literatura.

\section{MATERIAL Y MÉTODOS}

\section{LOCALIZACIÓN Y ANIMALES}

El trabajo de campo se desarrolló en 19 centros de producción, ubicados en 17 localidades y 8 comunidades de la Región de Huancavelica (tabla I), situadas en altitudes que oscilan entre 4000 y 4800 metros sobre el nivel del mar, con temperaturas que varían desde $-5^{\circ} \mathrm{C}$ a $0^{\circ} \mathrm{C}$. por las noches y durante el día entre 14 a $18^{\circ} \mathrm{C}$; y con una precipitación pluvial que alcanza los 752,4mm/año. 
Tabla I. Número de observaciones dentro de las localidades y comunidades, por sexo y edad. (Observations number within the localities and communities, by sex and age).

\begin{tabular}{|c|c|c|c|c|c|c|c|c|}
\hline \multirow[b]{2}{*}{ Comunidad } & \multirow[b]{2}{*}{ Localidad } & \multirow[b]{2}{*}{ Unidad productiva } & \multirow[b]{2}{*}{ Hembra } & \multirow[b]{2}{*}{ Macho } & \multicolumn{4}{|c|}{ Edad (años) } \\
\hline & & & & & $<1,5$ & $1,5-3$ & $3-4$ & $>4$ \\
\hline Carhuancho & Huaracco & CAR1 & 31 & 4 & 14 & 8 & 6 & 7 \\
\hline Carhuancho & Huaracco & CAR2 & 36 & 7 & 18 & 15 & 7 & 3 \\
\hline Carhuancho & Ccatunmachay & CAR3 & 35 & 9 & 20 & 14 & 8 & 2 \\
\hline Carhuancho & Accto & CAR4 & 28 & 0 & 11 & 3 & 13 & 1 \\
\hline Carhuancho & Pucachaca & CAR5 & 40 & 12 & 39 & 5 & 7 & 1 \\
\hline Carhuancho & Pucamachay & CAR6 & 20 & 0 & 5 & 2 & 11 & 2 \\
\hline Carhuancho & Pulchuhuasi & CAR7 & 24 & 9 & 27 & 0 & 1 & 5 \\
\hline Carhuancho & Cceccapallca & CAR8 & 11 & 1 & 1 & 2 & 9 & 0 \\
\hline Choclococha & Astohuarracca & $\mathrm{CHO} 1$ & 20 & 0 & 13 & 4 & 3 & 0 \\
\hline Choclococha & Champaccocha & $\mathrm{CHO} 2$ & 16 & 3 & 10 & 4 & 5 & 0 \\
\hline Choclococha & Choclococha & $\mathrm{CHO} 3$ & 20 & 1 & 5 & 7 & 9 & 0 \\
\hline Sallcca & Santa Ana & SAL1 & 28 & 1 & 13 & 6 & 9 & 1 \\
\hline Sallcca & Santa Ana & SAL2 & 17 & 2 & 1 & 11 & 7 & 0 \\
\hline Pucapampa & Pucapampa & PUC1 & 16 & 4 & 18 & 0 & 1 & 1 \\
\hline Pastales Huando & Jaccto & PAS1 & 10 & 1 & 10 & 1 & 0 & 0 \\
\hline Pastales Huando & Pucrocorral & PAS2 & 17 & 1 & 11 & 3 & 3 & 1 \\
\hline Sta. Bárbara & Yanacancha & SAN1 & 27 & 2 & 7 & 13 & 5 & 4 \\
\hline Lachocc & Tucumachay & UNH1 & 40 & 16 & 23 & 9 & 7 & 17 \\
\hline Alto Andino & Alto Andino & ALT1 & 22 & 13 & 26 & 2 & 4 & 3 \\
\hline
\end{tabular}

Los animales con que se trabajó estaban incluidos dentro del programa de mejoramiento genético de la región de Huancavelica, por lo cual tenían las características raciales fenotípicas de alpacas de la raza Huacaya, eran de color blanco entero y ninguna mostraba defectos genéticos.

\section{EVALUACIÓN DE LAS CARACTERÍSTICAS DE LA FIBRA}

Los datos de peso de vellón grasiento (PVG) fueron tomados de 544 alpacas, previamente identificadas con aretes de plástico sujetos a la oreja derecha, para luego registrarse el sexo y los dientes erupcionados a fin de determinar la edad $(<1,5$ años=dientes de leche; entre 1,5 a 3 años= dos incisivos permanentes; entre 3 y 4 años $=$ cuatro incisivos permanentes; $y>4$ años $=$ incisivos permanentes completos). Para la medición del diámetro de fibra(DF), coeficiente de variación del diámetro de fibra (CVDF) y factor de picazón (FP), se tomó una muestra del vellón de aproximadamente $20 \mathrm{~g}$ de la zona lateral central entre la línea superior e inferior del animal a la altura de la décima costilla, momentos antes de realizarse la esquila (Aylan-Parker y McGregor, 2002).

El PVG fue registrado inmediatamente después de la esquila, utilizando una balanza electrónica, de una sensibilidad de $5 \mathrm{~g}$; mientras que la medición del DF, CVDF y el FP se realizó en el Laboratorio de Fibras de la Universidad Nacional Agraria La Molina utilizando el Sirolan-Laserscan ${ }^{\mathrm{TM}}$ (CSIRO, Australia). Previamente a la medición se realizó el lavado de las muestras de fibra, primero con agua a $55^{\circ} \mathrm{C}$, luego con detergente industrial y carbonato de sodio en dosis de $2 \mathrm{~g} /$ litro de agua a $55^{\circ} \mathrm{C}$, posteriormente con detergente industrial y carbonato de sodio en dosis de $1 \mathrm{~g} /$ litro de agua a $55^{\circ} \mathrm{C}$ y finalmente con agua fría. Al culminar cada fase de lavado las muestras fueron exprimidas en un rodillo. Las muestras lava- 


\section{PROGRAMA DE MEJORA DE ALPACAS EN HUANCAVELICA-PERÚ}

das fueron secadas en una estufa a $105^{\circ} \mathrm{C}$, para posteriormente cardarlas a fin de darle orientación a las fibras y finalmente obtener cortes de $2 \mathrm{~mm}$ que fueron los que se introdujeron al Sirolan-Lasercan para la medición (IWTO, 1993).

La esquila se realizó mecánicamente a los animales que tenían una longitud de mecha $>$ a $9 \mathrm{~cm}$, y se adaptaron alzas a las esquiladoras mecánicas, que permitieron dejar una cubierta de fibra de unos $2 \mathrm{~cm}$, a fin de evitar dejar al animal sin ninguna cubierta de fibra, de modo que puedan tener cierta protección contra las bajas temperaturas de la zona. Las esquilas se realizaron en dos campañas (noviembre-diciembre y marzo-abril), luego de un periodo de crecimiento de la fibra no mayor de 2 años.

Los animales estuvieron sometidos a un sistema de crianza extensiva, pastoreando durante el día y por las noches eran recluidos en unos corrales hechos a base de piedra. La alimentación estuvo basada en pastos naturales, donde predominaban especies pertenecientes a las familias Poaceae Cyperaceae, Asteraceae y Juncaceae.

Para la evaluación de las características de la fibra se utilizó medias con su respectivo error estándar(E.E.). La determinación del efecto de los factores fijos: edad, sexo y locación sobre el PVG, DF, CVDF y FP, se hizo mediante un modelo aditivo lineal de efectos principales; en la comparación de medias se utilizó la prueba de Duncan; y la relación entre las variables de la fibra fue calculada mediante el coeficiente de Pearson. Se utilizó el programa informático SPSS Versión 12.

\section{Propuesta de un ESQuema de MEJORA Y PREDICCIÓNDELARESPUESTAALASELECCIÓN (RS)}

Para el planteamiento del esquema de mejora se tuvo en cuenta los índices productivos y reproductivos que se muestran en la tabla II, los cuales fueron encontrados a nivel de la región de Huancavelica por Nieto y Alejos (1999) y a nivel de Perú por
De Los Ríos (2006) y FAO (2005). El esquema de mejora a plantearse consideró la formación de un núcleo élite de reproductores machos, con los cuales se posibilitaría el empadre de las mejores reproductoras hembras, para producir crías machos suficientes para el reemplazo del grupo de reproductores machos con registros de producción a fin de empadrar la totalidad de hembras con registros.

Para la estimación del progreso genético o respuesta a la selección (RS), teniendo como criterio de selección el PVG y DF (caracteres más importantes en la comercialización y la industria de la fibra, de acuerdo a lo indicado por Wang et al., 2003; Frank et al., 2006; McGregor, 2006, Quispe, 2005), se consideraron las desviaciones estándar obtenidas en el presente estudio, mientras que las heredabilidades $\left(h^{2}\right)$, presión de selección (Ps) e intervalo generacional (I) fueron obtenidas de revisiones bibliográficas. Se consideraron heredabilidades de 0,30 y 0,25 para PVG y DF respectivamente,

Tabla II. Índices productivos y reproductivos considerados en el planteamiento del esquema de selección. (Production and reproduction indexes considered to establish the selection scheme).

\begin{tabular}{lc}
\hline Índice productivo & Valor \\
\hline Hembras/macho para empadre & 25 \\
Machos reemplazo de reproductores \% & 60 \\
Problemas en machos de reemplazo \% & 12 \\
Natalidad \% & 40 \\
Mortalidad (crías) \% & 10 \\
Mortalidad (adultos) \% & 5 \\
Machos de crías nacidas \% & 50 \\
Con registros de alpacas blancas \% & 10 \\
No producen fibra (de alpacas blancas) $\%$ & 30 \\
& \\
Composición de la población de alpacas blancas \\
con registros (expresado en \%) \\
$\quad$ Crías (machos y hembras) \\
Tuis (< de 3 años) \\
$\quad$ Machos reproductores \\
Hembras reproductoras & 30,0 \\
& 28,0 \\
\end{tabular}


en un escenario pesimista y de 0,41 y 0,37 para PVG y DF respectivamente en un escenario optimista .La presión de selección y el intervalo generacional se asumieron bajo dos posibles escenarios: pesimista $\left(\mathrm{Ps}_{\mathrm{s}}=0,07\right.$ y 0,$21 ; \mathrm{I}=5,5$ y 6,0 ; para machos y hembras respectivamente) y optimista $\left(\mathrm{Ps}_{\mathrm{s}}=0,04 \mathrm{y}\right.$ 0,11 ; I $=4,5$ y 5,5 ; para machos y hembras respectivamente). La RS se calculó para la selección de cada carácter por separado y combinando ambos en un agregado económico. El cálculo de la RS para cada carácter por separado se predijo según la ecuación:

$$
R S=\left(i^{*} h^{2 *} S_{p}\right) / l,
$$

donde:

$\mathrm{i}=$ intensidad,

$\mathrm{h}^{2}=$ heredabilidad

$\mathrm{s}_{\mathrm{p}}=$ desviación estándar fenotípica,

I= intervalo generacional.

Para la predicción de RS en unidades monetarias $(\$)$ se consideró un precio de \$ 7,00 US por $\mathrm{kg} /$ fibra, un costo de producción de $\$ 3,5 \mathrm{US} / \mathrm{kg}$ de fibra producida y que por la disminución en $1 \mu \mathrm{m}$ en un vellón que pesa $2300 \mathrm{~g}$ se obtiene a la venta un adicional de \$2,38 US. Los pesos económicos utilizados para el cálculo de los índices de selección (con y sin restricción) del PVG $\left(a_{\mathrm{PVG}}=0,0137\right)$ y del DF $\left(\mathrm{a}_{\mathrm{DF}}=-2,375\right)$ fueron calculados en base a una derivación parcial de la función de beneficio (ingresos-costes), a fin de maximizar ganancias (Quispe y Alfonso, 2007); mientras que la correlación fenotípica entre PVG y DF fue estimada a partir de los datos, y para la correlación genotípica se consideraron diversos valores a fin de asumir múltiples escenarios. Para la predicción del RS anual se utilizó la siguiente ecuación matricial:

$$
R S=\frac{\sqrt{b^{* *} P^{*} b}}{b^{*} G} * \frac{i}{I},
$$

donde:

$b=$ matriz de coeficientes del índice de selección, $b^{\prime}=b$ transpuesta,

$\mathrm{P}=$ matriz de varianzas-covarianzas fenotípicas,
$\mathrm{G}=$ matriz de varianzas-covarianzas genéticas de PVG y DF,

I= intervalo generacional.

\section{RESULTADOSYDISCUSIÓN}

\section{EVALUACIÓN DE LAS CARACTERÍSTICAS DE LAFIBRA}

Las medias \pm E.E. obtenidas para $P V G$, DF, CVDF y FP fueron de $2300 \pm 39$ g, 21,56 $\pm 0,12 \mu \mathrm{m}, 22,82 \pm 0,12 \%$ y $6,33 \pm 0,30 \%$ respectivamente. La media general del PVG resulta ser mayor a los valores reportados por Jáuregui y Bonilla (1991), Castellaro et al. (1998), Wuliji et al. (2000), León-Velarde y Guerrero (2001); similar a lo encontrado por Condorena (1985), Bryant et al. (1989), Nieto y Alejos (1999) y De Los Ríos (2006), aunque resulta ser menor a lo reportado por Ponzoni (1999), Ponzoni (2000), McGregor (2002) y McGregor (2006), pudiendo deberse esta superioridad a que dichos animales estuvieron bajo condiciones de una mejor alimentación la cual tiene efecto positivo en la producción de la fibra (Bryant et al., 1989), contrario a una nutrición inadecuada lo cual disminuye el crecimiento de la fibra, tal como es discutido por McGregor (2002). Por otro lado el PVG exhibe una regular variabilidad (coeficiente de variación= $39,44 \%$ ), lo que justificaría la inclusión de dicho criterio para programas de mejora genética, toda vez que también constituye un factor de importancia al momento de la comercialización del vellón.

El DF es menor a los reportados por Ponzoni et al. (1999), Wuliji et al. (2000), Wang et al. (2003), que conlleva a considerar que un gran porcentaje de vellones que se producen en estos rebaños corresponderían un fibra fina $(20,1$ a $23 \mu \mathrm{m})$ y superfina $(<20 \mu \mathrm{m})$, contrario a lo indicado por De los Ríos (2006) y McGregor (2006), lo cual indicaría la existencia de animales con buena calidad de fibra que podría hacer factible la formación de una élite de reproductores para poder difundir adecuadamente este deseable potencial genético; sin embargo 


\section{PROGRAMA DE MEJORA DE ALPACAS EN HUANCAVELICA-PERÚ}

esta aparente finura podría deberse a las condiciones pobres de alimentación de los animales en estudio (bajo pasturas naturales), lo cual concordaría a lo indicado por McGregor (2002) y Marshall et al. (1981), y corroborado por Wuliji et al. (2000), quienes demostraron que las condiciones nutricionales adversas reducen el DF, contrario al efecto que causa una buena alimentación, lo cual incrementa el diámetro de fibra.

La media del CVDF obtenida, que resultaría ser bastante buena para las exigencia de la industria textil, es ligeramente inferior a lo obtenido por Aylan-Parker y McGregor (2002), Wang et al. (2003), McGregor y Butler (2004) y Lupton et al. (2006), quienes reportan valores entre 23,48 y $28,10 \%$. Esta característica tiene alta influencia sobre algunas propiedades requeridas en la industria textil (Lupton et al., 2006), pues conjuntamente con el DF determinan la finura para el torcido de la fibra asociado al rendimiento del hilado, propiedad conocida también como spinning finnes (SF), cuyo cálculo está basado en el DF con un CVDF de $24 \%$, de modo que valores debajo de ello resultan en un SF más bajo que el DF y valores por encima de ello resultan en un SF más alto (McGregor, 2006), debido a que cuando se tienen fibras de diámetro heterogéneo, se requieren mayor número de ellas para alcanzar igual resistencia y uniformidad (Mueller, 2000). Por otro lado, el FP está asociado a la sensación de picazón (prickle factor), por lo que la industria textil requiere de vellones con FP muy bajos. La media \pm E.E. del FP encontrada $(6,33 \pm 0,30 \%)$ resulta ser muy inferior a $31,61 \%$, valor que es reportado por Lupton et al. (2006), lo cual se debería al valor alto de DF que encuentra.

El PVG y el DF muestran variaciones altamente significativas $(\mathrm{p}<0,01)$ para sexo, edad y unidad productiva, resultando que animales jóvenes tienen menor PVG y DF en contraste con los de mayor edad, mientras que las hembras tienen menor PVG y mayor DF que los machos (tabla III).

Las variaciones del PVG por efecto de la edad y el sexo concuerdan a lo reportado por Castellaro et al. (1998), Wuliji et al. (2000), McGregor (2006) y Lupton et al., (2006), de modo que el mayor PVG de los animales adultos se debería al incremento de la superficie corporal (León-Velarde y Guerrero, 2001; Frank et al., 2006), y la influencia del sexo sería debida a que la preñez y la lactación de la hembras reducirían la producción (Newman y Paterson, 1996).

La variación del DF por efecto de la edad concuerda a lo hallado por Wuliji et al. (2000), quien reporta medias de DF de 26,5 y $32,0 \mu \mathrm{m}$ para crías y adultos respectivamente, lo cual también está acorde a lo encontrado por Lupton et al. (2006). McGregor y Butler (2004), en función a la diferencia de DF que encuentra en alpacas de distintas edades posibilita la existencia del micron blowout, término que es utilizado en la industria lanar para describir el incremento del diámetro de la lana con la edad que no es debido a influencias medioambientales temporales. Por otro lado, el efecto del sexo sobre el DF encontrado resulta contradictorio a lo reportado por Lupton et al. (2006) y Aylan-Parker y McGregor (2002), quienes indican que los machos tienen mayor DF que las hembras; y también difiere a lo encontrado por Wuliji et al. (2000), McGregor y Butler (2004) y Frank et al. (2006), quienes manifiestan que no existe efecto del sexo sobre el DF; sin embargo nuestros resultados resultan similares a lo encontrado por Montes et al. (2008). La causas más probables de estos hallazgos serían que los criadores realizan una selección subjetiva de machos mucho más minuciosa que las hembras, y que las hembras generalmente provienen de la misma región de Huancavelica, mientras que los machos tienen orígenes de otras regiones del territorio peruano (Puno y Cusco principalmente).

El FP tiene variaciones altamente significativas $(p<0,01)$ para edad y unidad de producción (tabla III). Los animales adultos ( $>$ de 4 años) exhiben mayor FP que los 


\section{QUISPE, ALFONSO, FLORES, GUILLÉNY RAMOS}

animales jóvenes ( $<$ de 1,5 años).

Por otro lado, la falta de efecto del sexo sobre el CVDF, concuerda con lo reportado por McGregor y Butler (2004), sin embargo la falta de efecto de la edad sobre CVDF, resulta discordante con lo hallado por los investigadores antes citados, quienes en- cuentran evidencia que el CVDF disminuye rápidamente hasta los 2 a 3 años y para luego incrementar levemente hasta los 10 años.

Los resultados de la variabilidad del FP por efecto de la edad, resulta concordante a lo reportado por Lupton et al. (2006), asi-

Tabla III. Medias \pm error estándar (EE) de peso de vellón grasiento ( $P V G)$, diámetro de fibra $(D F)$, coeficiente de variación del diámetro de fibra (CVDF) y factor de picazón (FP) por sexo, edad y unidad productiva (UP). (Means \pm standard error (EE) of grease fleece weight (PVG), fibre diameter (DF), coefficient of variation of fiber diameter (CVDF) and prickle factor (FP) by sex, age and unit productive (UP)).

\begin{tabular}{|c|c|c|c|c|c|}
\hline Factor de estudio & $\mathrm{n}$ & $\mathrm{PVG}^{1}(\mathrm{~g})$ & $\mathrm{DF}^{1}(\mu \mathrm{m})$ & $\operatorname{CVDF}^{1}(\%)$ & $\mathrm{FP}^{1}(\%)$ \\
\hline Sexo* & & {$[0,007]$} & {$[0,007]$} & {$[0,189]$} & {$[0,109]$} \\
\hline Hembra & 458 & $2272 \pm 41^{a}$ & $21,66 \pm 0,13^{b}$ & $22,74 \pm 0,13$ & $6,50 \pm 0,34$ \\
\hline Macho & 86 & $2450 \pm 112^{b}$ & $21,00 \pm 0,26^{a}$ & $23,21 \pm 0,30$ & $5,42 \pm 0,59$ \\
\hline Edad $^{*}$ & & {$[0,000]$} & {$[0,000]$} & {$[0,110]$} & {$[0,000]$} \\
\hline Dientes de Leche & 272 & $2006 \pm 44^{a}$ & $20,75 \pm 0,15^{a}$ & $23,12 \pm 0,18$ & $4,66 \pm 0,29^{a}$ \\
\hline Dos dientes & 109 & $2420 \pm 76^{b}$ & $21,67 \pm 0,24^{b}$ & $22,56 \pm 0,26$ & $6,20 \pm 0,58^{a}$ \\
\hline Cuatro dientes & 115 & $2668 \pm 87^{c}$ & $22,75 \pm 0,26^{c}$ & $22,51 \pm 0,25$ & $8,97 \pm 0,88^{b}$ \\
\hline Boca llena & 48 & $2818 \pm 191^{c}$ & $23,00 \pm 0,44^{c}$ & $22,41 \pm 0,38$ & $9,75 \pm 1,38^{b}$ \\
\hline$U^{*}$ & & {$[0,000]$} & {$[0,000]$} & {$[0,000]$} & {$[0,000]$} \\
\hline CAR1 & 35 & $1579 \pm 87^{a b}$ & $23,12 \pm 0,62^{f}$ & $23,85 \pm 0,63^{\text {def }}$ & $11,88 \pm 2,12^{g}$ \\
\hline CAR2 & 43 & $2489 \pm 98^{\text {ef }}$ & $23,35 \pm 0,44^{f}$ & $24,24 \pm 0,45^{f}$ & $10,56 \pm 1,34^{\text {efg }}$ \\
\hline CAR3 & 44 & $3005 \pm 144^{9}$ & $21,36 \pm 0,36^{\text {cde }}$ & $22,04 \pm 0,33^{\mathrm{bcd}}$ & $5,43 \pm 1,21^{\mathrm{abcd}}$ \\
\hline CAR4 & 28 & $2905,12 \pm 112^{\mathrm{fg}}$ & $22,04 \pm 0,42^{\text {def }}$ & $22,17 \pm 0,44^{\text {bcd }}$ & $6,23 \pm 0,82^{\mathrm{abcd}}$ \\
\hline CAR5 & 52 & $1962,97 \pm 74^{\text {bcd }}$ & $21,01 \pm 0,28^{\text {bcde }}$ & $22,32 \pm 0,33^{\mathrm{bcd}}$ & $5,03 \pm 0,69^{a b c d}$ \\
\hline CAR6 & 20 & $3211 \pm 159^{9}$ & $22,20 \pm 0,60^{\mathrm{ef}}$ & $22,00 \pm 0,55^{\mathrm{bc}}$ & $6,61 \pm 1,33^{\text {abcde }}$ \\
\hline CAR7 & 33 & $1871 \pm 48^{\text {bcd }}$ & $19,92 \pm 0,31^{a b c}$ & $24,51 \pm 0,43^{f}$ & $3,71 \pm 0,43^{\text {abcd }}$ \\
\hline CAR8 & 12 & $2812 \pm 184^{\mathrm{fg}}$ & $22,32 \pm 0,64^{e f}$ & $24,16 \pm 0,55^{e f}$ & $7,69 \pm 1,54^{\mathrm{def}}$ \\
\hline $\mathrm{CHO} 1$ & 20 & $1280 \pm 66^{a}$ & $20,32 \pm 0,63^{a b c}$ & $23,63 \pm 0,55^{\text {bcdef }}$ & $4,58 \pm 0,93^{\mathrm{abcd}}$ \\
\hline $\mathrm{CHO} 2$ & 19 & $1942 \pm 169^{\mathrm{bcd}}$ & $19,11 \pm 0,41^{a}$ & $22,36 \pm 0,64^{\text {bcde }}$ & $2,29 \pm 0,33^{a}$ \\
\hline $\mathrm{CHO} 3$ & 21 & $2230 \pm 124^{\text {de }}$ & $21,16 \pm 0,53^{\text {cde }}$ & $22,14 \pm 0,65^{\mathrm{bcd}}$ & $4,58 \pm 0,70^{\text {abcd }}$ \\
\hline PUC1 & 20 & $1330 \pm 106^{a}$ & $22,03 \pm 0,65^{\text {def }}$ & $22,91 \pm 0,63^{\text {bcdef }}$ & $6,98 \pm 1,63^{\text {bcde }}$ \\
\hline SAN1 & 29 & $2314 \pm 148$ & $19,99 \pm 0,40^{\mathrm{abc}}$ & $20,16 \pm 0,44^{a}$ & $2,61 \pm 0,49^{a b}$ \\
\hline SAL1 & 29 & $2505 \pm 130^{\mathrm{ef}}$ & $20,54 \pm 0,42^{\mathrm{abcd}}$ & $23,19 \pm 0,36^{\text {bcdef }}$ & $4,49 \pm 0,77^{\mathrm{abcd}}$ \\
\hline SAL2 & 19 & $2002 \pm 149^{\mathrm{bcd}}$ & $23,33 \pm 0,73^{f}$ & $23,78 \pm 0,72^{\text {cdef }}$ & $11,11 \pm 2,88^{\mathrm{fg}}$ \\
\hline PAS1 & 11 & $2124 \pm 209^{\text {cde }}$ & $19,58 \pm 0,51^{\mathrm{ab}}$ & $23,73 \pm 0,46^{\text {bcdef }}$ & $2,99 \pm 0,50^{a b c}$ \\
\hline PAS2 & 18 & $1941 \pm 95^{\mathrm{bcd}}$ & $21,42 \pm 0,55^{\text {cde }}$ & $22,39 \pm 0,64^{\text {bcde }}$ & $5,26 \pm 1,24^{\mathrm{abcd}}$ \\
\hline ALT1 & 35 & $1746 \pm 79^{\mathrm{bc}}$ & $22,26 \pm 0,40^{\text {ef }}$ & $21,93 \pm 0,63^{b}$ & $6,86 \pm 1,13^{\text {bcde }}$ \\
\hline UNH1 & 56 & $3228 \pm 155^{9}$ & $22,18 \pm 0,33^{\text {ef }}$ & $22,94 \pm 0,27^{\text {bcdef }}$ & $7,06 \pm 0,67^{\text {cde }}$ \\
\hline Global & 544 & $2300 \pm 39$ & $21,56 \pm 0,12$ & $22,82 \pm 0,12$ & $6,33 \pm 0,30$ \\
\hline
\end{tabular}

*Entre corchetes se encuentran los p-valores que evalúan la significación de los efectos de sexo, edad y unidad productiva sobre las variables de la fibra. n: Número de animales. ${ }^{1}$ Medias, dentro de un efecto dado y para cada variable, con diferentes letras muestran diferencias significativas $(p<0,05)$ a la prueba de Duncan.

Archivos de zootecnia vol. 58, núm. 224, p. 712. 


\section{PROGRAMA DE MEJORA DE ALPACAS EN HUANCAVELICA-PERÚ}

mismo la falta de variabilidad del FP por efecto del sexo encontrada, resulta concordante con Lupton et al. (2006) cuando compara FP entre alpacas machos y hembras.

Las variaciones de todas las características de la fibra en estudio (PVG, DF, CVDF e FP) debido al efecto de las unidades productivas, resultan justificadas debido a que cada una de ellas pertenecen a diferentes propietarios y se encuentran localizados en diversos lugares de la región (Huancavelica), lo que hace que se encuentren sometidos a diferentes prácticas de manejo (alimentación, sanidad, entre otros) y que también podría estar influyendo el origen genético (Montes et al., 2008), por lo que convendría el ingreso de este factor fijo dentro de un modelo de evaluación genética.

Las correlaciones entre DF y FP, DF y PVG, y CVDF y FP son altamente significativas $(\mathrm{p}<0,01)$, sin embargo sólo la primera resulta ser alta $(0,844)$, mientras que las otras son muy bajas $(0,112$ y 0,139 respectivamente). La correlación entre CVDF y DF es sólo significativa $(<0,05)$, pero el valor es bajo (-0,088). Finalmente las correlaciones entre PVG con CVDF y FP no son significativas (tabla IV).

Aunque las correlaciones fenotípicas pueden ser buenos indicadores de la magnitud y del signo de las correlaciones genéticas, resulta necesario realizar trabajos de investigación en torno a ellas, lo cual marcaría la dirección adecuada de las formas de selección a utilizarse. La existencia de relación encontrada entre PVG y DF es baja frente a correlaciones de 0,$25 ; 0,45 \mathrm{y}$ $0,53(p<0,01)$ determinadas en rebaños de Australia, Nueva Zelanda y Norteamérica por Ponzoni et al. (1999), Wuliji et al. (2000) y Lupton et al. (2006) respectivamente; sin embargo, esta débil relación podría deberse al amplio rango de intervalo entre esquilas que tuvieron las alpacas en estudio (los animales fueron esquilados en base a una longitud de mecha $=9 \mathrm{~cm}$ ), y que también factores como altitud, condición alimenti-
Tabla IV. Correlaciones fenotipicas entre peso de vellón grasiento $(P V G)$, diámetro de fibra $(D F)$, coeficiente de variación del diámetro de fibra (CVDF) y factor de picazón $(F P)$. (Phenotipic correlations between grease fleece weight (PVG), fibre diameter (DF), coefficient of variation of fiber diameter (CVDF) and prickle factor (FP)).

\begin{tabular}{lcccc}
\hline Variables & $n$ & PVG & DF & CVDF \\
\hline DF & 544 & $0,112^{* *}$ & & \\
CVDF & 544 & $-0,80$ & $-0,088^{*}$ & \\
FP & 544 & 0,045 & $0,844^{* *}$ & 0,139 \\
\hline
\end{tabular}

$n$ : número de observaciones. ${ }^{*} p<0,05 ;{ }^{* *} p<0,01$.

cia, condición corporal, entre otros, podrían estar influyendo.

La débil relación entre CVDF con PVG y FD, concuerda con lo reportado por Ponzoni et al. (1999), mientras que la falta de relación encontrada entre PVG y FP, así como la baja relación entre DF y CVDF, no han sido bien estudiadas en esta especie, aunque Safari et al. (2005) al realizar una revisión de 10 investigaciones en ovinos obtiene un coeficiente de correlación entre DF y CVDF de -0,09, el cual resulta casi nulo, la que guardaría concordancia con el resultado de este trabajo. Por otro lado, la alta relación encontrada entre DF y FP, se hace evidente, ya que cuanto menor sea el DF existirían también menos cantidad de fibras que estén por encima de las $30 \mu \mathrm{m}$.

\section{ESQUEMADE MEJORA YLARESPUESTAALA SELECCIÓN(RS)}

El esquema de mejora planteado tiene como base una población inicial de alpacas blancas $(\approx 193500)$ correspondiente a la región de estudio, de la cual se toma una fracción $(10 \%)$ para el monitoreo de registro de datos de producción y genealógicos (Ponzoni, 2000), y se llega a un núcleo de 50 machos reproductores, que con cubrición natural, podría empadrar a unas 1110 hembras seleccionadas, que bajo las tasas de 


\section{QUISPE, ALFONSO, FLORES, GUILLÉNY RAMOS}

nacimiento y mortalidad propios de la zona producirán la suficiente cantidad de machos de reemplazo para servir a las hembras en control, de modo que los machos que son reemplazados producirán el flujo de genes hacia la población de animales sin registros (majada).

Este esquema de selección utilizado en un programa de mejoramiento genético de núcleo abierto posibilita su aplicación dentro de un entorno colectivo, lo cual se hace conveniente en la región de estudio, donde los productores tienen hatos conformados por pocos animales (entre 70 a 150 animales), siendo necesario para ello el acuerdo entre los productores (Mueller, 2003), quienes tendrían un rol decisivo para alcanzar los objetivos deseados.

Los resultados de las predicciones de la RS anual, en magnitudes de los criterios de selección ( $\mathrm{PVG}$ en $\mathrm{g}$ y $\mathrm{DF}$ en $\mathrm{mm}$ ) y en unidades monetarias por animal (US\$/a), cuando se seleccionan independientemente por producción individual de PVG o DF, así como cuando se seleccionan en conjunto por las producciones de PVG y DF utilizando índices de selección sin restricción encontrados $(I=0,0026 * P V G-0,0020 * D F$ y $I=0,0035^{*} P V G-0,1100^{*} D F$ para escenarios pesimista y optimista respectivamente) y con restricción para $\mathrm{PVG}$ encontrados $(I=0,0007 * P V G-0,3660 * D F$ y $I=0,0003 *$ $P V G-0,8820^{*} D F$ para escenarios pesimista y optimista respectivamente), se muestran en la tabla $\mathbf{V}$, observándose un mejor retorno económico (\$1,16 US/animal/año) cuando la selección se realizaría utilizando un índice de selección sin restricción en un escenario optimista si la correlación genética fuese baja $\left(\mathrm{r}_{\mathrm{G} \text { (PVG }}=0,05\right)$ y un bajo retorno económico ( 0,11 ÚS \$ /animal/año) cuando la selección se realizaría utilizando un índice

Tabla $\boldsymbol{V}$. Estimaciones de la respuesta a la selección $(R S)$ por año, obtenidas bajo dos escenarios, para peso de vellón grasiento $(P V G)$ y diámetro de fibra (DF) por selección masal y usando un índice de selección para su agregado económico, bajo correlaciones genéticas entre PVG y DF bajas $(0,05)$ y altas $(0,65)$. (Estimation of the genetic progress (RS) for year, obtained under two scenarios, for grease fleece weight (PVG) and fibre diameter (DF) using individual selection and selection index for the joint economic value, with genetic correlations between PVG and DF, low (0.05) and great (0.65)).

\begin{tabular}{|c|c|c|c|c|c|c|c|}
\hline \multirow[b]{2}{*}{ Tipo de selección } & \multirow[b]{2}{*}{ Escenario } & \multirow{2}{*}{$\begin{array}{c}\text { Correlación } \\
\text { genotípica } \\
\mathrm{r}_{(\mathrm{PVG}, \mathrm{df})}\end{array}$} & \multicolumn{2}{|c|}{ RS/año } & \multicolumn{3}{|c|}{ RS/año } \\
\hline & & & $\begin{array}{c}\text { PVG } \\
\mathrm{g}\end{array}$ & $\begin{array}{l}\text { DF } \\
\mu \mathrm{m}\end{array}$ & $\begin{array}{l}\text { PVG } \\
\text { US\$ }\end{array}$ & & $\begin{array}{c}\text { DF } \\
\text { US\$ }\end{array}$ \\
\hline \multirow[t]{2}{*}{ Masal } & Pesimista & - & 56,13 & $-0,17$ & 0,39 & & 0,40 \\
\hline & Optimista & - & 109,13 & $-0,35$ & 0,74 & & 0,83 \\
\hline \multirow[t]{4}{*}{ Índice sin restricción } & Pesimista & 0,05 & 49,77 & $-0,08$ & \multicolumn{3}{|c|}{0,54} \\
\hline & Pesimista & 0,65 & 55,73 & 0,12 & \multicolumn{3}{|c|}{0,11} \\
\hline & Optimista & 0,05 & 97,64 & $-0,20$ & \multicolumn{3}{|c|}{1,16} \\
\hline & Optimista & 0,65 & 103,25 & 0,21 & \multicolumn{3}{|c|}{0,23} \\
\hline \multirow[t]{4}{*}{ Índice con restricción de PVG } & Pesimista & 0,05 & 0,00 & $-0,17$ & \multicolumn{3}{|c|}{0,40} \\
\hline & Pesimista & 0,65 & 0,00 & $-0,09$ & \multicolumn{3}{|c|}{0,21} \\
\hline & Optimista & 0,05 & 0,00 & $-0,35$ & \multicolumn{3}{|c|}{0,84} \\
\hline & Optimista & 0,65 & 0,00 & $-0,18$ & \multicolumn{3}{|c|}{0,44} \\
\hline
\end{tabular}

$\mathrm{I}=$ Intervalo generacional $=5,75$ y 4,75 (escenario pesimista y optimista respectivamente); $\mathrm{i}=$ intensidad de selección= 1,65 y 1,93 (escenario pesimista y optimista respectivamente); RS= Respuesta a la selección.

Archivos de zootecnia vol. 58, núm. 224, p. 714. 


\section{PROGRAMA DE MEJORA DE ALPACAS EN HUANCAVELICA-PERÚ}

de selección sin restricción en un escenario pesimista si la correlación genética fuese $\operatorname{alta}\left(\mathrm{r}_{\mathrm{G}(\mathrm{PVG}, \mathrm{DF})}=0,65\right)$.

Existen pocos trabajos referidos a estimaciones de progreso genético en alpacas de color blanco, y prácticamente es nula la información para zonas alto andinas. Nuestras predicciones de RS anual son menores a las de Ponzoni (2000), en un estudio realizado en Australia, lo cual se debería a los valores económicos utilizados, para peso de vellón y diámetro de fibra, más altos que los nuestros $(15,00$ y 3,00 US $\$$ contra 7,00 y 2,38 US\$ respectivamente).

Uno de los inconvenientes para la predicción de RS resulta ser la ausencia de información sobre parámetros genéticos en alpacas a nivel de la sierra central, pero es más alarmante la falta de reportes sobre correlaciones genéticas a nivel mundial. Bajo estas consideraciones, la estimación

\section{BIBLIOGRAFÍA}

Antonini, M., A. Gonzáles and A. Valbonise. 2004. Relationship between age and postnatal skin follicular development in three types of South American domestic camelids. Livest. Prod. Sci., 90: 241-246.

Apomayta, Z. y G. Gutiérrez. 1998. Evaluación de características tecnológicas y productivas de la fibra en alpacas Huacaya esquiladas a los 12 y 17 meses de edad. Anales Científicos. UNA, 36: 35-42.

Aylan-Parker, J. and B.A. McGregor. 2002. Optimising sampling techniques and estimating sampling variance of fleece quality attributes in alpacas. Small Ruminant Res., 44: 53-64.

Braga, W., V. Leyva and R. Cochran. 2006. The effect of altitude on alpaca (Lama pacos) fiber production. Technical note. Small Ruminant Res., 68: 323-328.

Bryant, F.C., A. Florez and J. Pfister. 1989. Sheep and alpaca productivity on high andean rangelands in Peru. J. Anim. Sci., 67: 3078-3095.

Castellaro, G., J. Garcia-Huidobro and P. Salinas. 1998. Alpaca liveweight variations and fiber production in Mediterranean range of Chile. J. Range Manage., 51: 509-513. de correlaciones genéticas en alpacas se hace urgente pues servirá para plantear formas adecuadas de selección que permitan obtener los más altos progresos genéticos posibles, lo cual conllevaría a la mejora de ingresos económicos para el productor alpaquero.

\section{AGRADECIMIENTOS}

Agradecemos a todos los propietarios de las diferentes unidades de producción, quienes nos permitieron tomar la información, ejecutar la esquila y obtener muestras de sus rebaños. También apreciamos la asistencia financiera de Dirección Universitaria de Investigación de la Universidad Nacional de Huancavelica y de INCAGRO (Contrato $\mathrm{N}^{\circ}$ 2006-00211), institución dependiente del Ministerio de Agricultura del Gobierno de Perú.

Chávez, J. y A. Pumayalla. 1988. Efectos de localidad, edad y sexo sobre el peso de vellón en alpacas Huacaya de una SAIS de Puno. VI Conv. Int. de Especialistas en Cam., Oruro, Bolivia.

Condorena, N. 1985. Aspectos de un sistema regularizador de la crianza de alpacas. IVITA La Raya. Puno, Perú.

De los Ríos, E. 2006. Producción textil de fibras de camélidos sudamericanos en el área altoandina de Bolivia, Ecuador y Perú. Organización de las Naciones Unidas para el Desarrollo Industrial (UNIDO). https://www.unido.org/file-storage/ download/?file_id=58563. [26 de septiembre del 2007]

FAO. 2005. Situación actual de los camélidos Sudamericanos en el Perú. Roma.

Frank, E.N., M.V.H. Hick, C.D. Gauna, H.E. Lamas, C. Reniere and M. Antonini. 2006. Phenotypic and genetic description of fibre traits in South American domestic camelids (Ilamas and alpacas). Small Ruminant Res., 61: 113-129.

Hoffman, E. and M.E. Fowler. 1995. The alpaca book. Clay Press Inc., Herald, California.

IWTO. 1993. Measurement of the mean and 


\section{QUISPE, ALFONSO, FLORES, GUILLÉNY RAMOS}

distribution of fiber diameter using the SirolanLasersacan Fiber Diameter Analyzer. IWTO Test Method, pp. 12-93.

Jaúregui, V. y G. Bonilla. 1991. Productividad de carne, fibra y cuero en alpacas y llamas. XIV Reunión Científica APPA. Cerro de Pasco. Perú.

León, U. 1998. Efecto del ambiente controlado y no controlado sobre el diámetro de lana y fibra de alpaca en verano e invierno. Tesis UNA La Molina. Facultad de Zootecnia. Lima, Perú.

León-Velarde, C.U. and J. Guerrero. 2001. Improving quantity and quality of alpaca fiber; using simulation model for breeding strategies. http://inrm.cip.cgiar.org/home/publicat/ 01cpb023.pdf. [24 de septiembre de 2007].

Lupton, C.J., A. McColl and R.H. Stobart. 2006. Fiber characteristics of the Huacaya Alpaca. Small Ruminant Res., 64: 211-224.

Mamani, G. 1991. Parámetros genéticos del peso vivo y vellón en alpacas Huacaya de La Raya, Puno. En: VII Conv. Int. Especialistas en Cam. Sud., Jujuy, Argentina.

Marshall, A.J., V. Bustinza y T.L. Quispe. 1981. Efecto de la alimentación con alfalfa sobre la producción y reproducción de la alpaca. V Reunión Científica APPA. Ayacucho, Perú.

McGregor, B.A. 2002. Comparative productivity and grazing behaviour of Huacaya alpacas and peppin Merino sheep grazed on annual pastures. Small Ruminant Res., 44: 219-232.

McGregor, B.A. and K.L. Butler. 2004. Sources of variation in fibre diameter attributes of Australian alpacas and implications for fleece evaluation and animal selection. Aust. J. Agr. Res., 55: 433442.

McGregor, B.A. 2006. Production, attributes and relative value of alpaca fleeces in southern Australia and implications for industry development. Small Ruminant Res., 61: 93-111.

Montes, M., I. Quicaño, R. Quispe, E.C. Quispe and L. Alfonso. 2008. Quality characteristics of Huacaya Alpaca fibre produced in the Peruvian Andean Plateau region of Huancavelica. Span. J. Agr. Res., 6: 33-38.

Mueller, J. 2000. Mejoramiento genético de la lana. Caracteres de importancia. http://www.inta. gov.ar/bariloche/info/documentos/animal/ genetica/pa374.pdf. [1 de octubre de 2007].
Mueller, J. 2003. Estrategías para el mejoramiento genético de pequeños rumiantes. http://www. inta.gov.ar/bariloche/info/documentos/animal/ genetica/pa425.pdf. [9 de noviembre de 2007].

Newman, S-A. N. and D.J. Paterson. 1996. Estimates of environmental effects for liveweight and fleece characteristics of New Zealand cashmere goats. N. Z. J. Agr. Res., 39: 379-386.

Nieto, L. e I. Alejos. 1999. Estado económico y productivo del Centro de Producción e Investigación de Camélidos Sudamericanos. Lachocc. XXI Reunión Científica Anual APPA. Huancavelica, Perú.

Ponzoni, R.W., R.J. Grimson, J.A. Hill, D.J. Hubbard, B.A. McGregor, A. Howse, I. Carmichael and G.J. Judson. 1999. The inheritance of and association among some production traits in young Australian alpacas. P. Aust. Assoc. Anim. Breed. Genet., 13: 468-471. http://www. alpacas.com/. [18 de septiembre de 2007].

Ponzoni, R.W. 2000. Genetic improvement of Australian Alpacas: present state and potencial developments. Proc. Aust. Alpaca Assoc., 1: 71-96.

Quispe, E.C. 2005. Mejoramiento genético y medioambiental de alpacas en la región de Huancavelica. Proyecto de inversión pública a nivel de perfil. Universidad Nacional de Huancavelica. Perú.

Quispe, E.C. y L. Alfonso. 2007. Metodología para estimar valores de cría: Aplicaciones para el mejoramiento genético de alpacas. Ediciones UNH. Huancavelica. Perú.

Safari, E., N.M. Fogarty and A.R. Gilmour. 2005. A review of genetic parameter estimates for wool, growth, meat and reproduction traits in sheep. Livest. Prod. Sci., 92: 271-289.

Wang, X., L. Wang and X. Liu. 2003. The quality and processing performance of alpaca fibres: Australian alpaca fibre industry and the fibre properties. http://www.rirdc.gov.au/reports/ RNF/03-128.pdf. [25 de septiembre de 2007]. Wuliji, T., G.H. Davis, K.G. Dodds, P.R. Turner, R.N. Andrews and G.D. Bruce. 2000. Production performance, repeatability and heritability estimates for live weight, fleece weight and fiber characteristics of alpacas in New Zealand. Small Ruminant Res., 37: 189-201. 